\title{
Can we prevent deaths of homeless persons? Police led public health approach to prevent homeless deaths
}

\author{
Natalie Kroovand Hipple* \\ Indiana University \\ Sarah J. M. Shaefer \\ National Fetal and Infant Mortality Review Program \\ American College of Obstetricians and Gynecologists \\ Robert F. Hipple, Jr. \\ Indianapolis Metropolitan Police Department
}

Alfarena T. Ballew

Chief Deputy Coroner

September 2016

*Corresponding author:

Natalie Kroovand Hipple

Assistant Professor

Indiana University

1033 East Third Street

309 Sycamore Hall

Bloomington, IN 47405

nkroovan@indiana.edu (e)

812.856 .2759 (o)

$812.855 .5522(\mathrm{f})$

The Version of Record of this manuscript has been published and is available in The Journal of Social Distress and the Homeless, October 14, 2016 http://tandfonline.com/10.1080/10530789.2016.1240950 


\begin{abstract}
Research on homeless populations demonstrates that homelessness in itself is an independent risk factor for death. However, there is a dearth of detailed data on homeless decedents and the situations surrounding their deaths. This lack of knowledge, a desire to understand how and why homeless individuals were dying, and a sentinel event death led the Indianapolis Metropolitan Police Department Homelessness and Panhandling Unit to partner with a local researcher and begin conducting homeless death reviews. The approach is modeled after the evidence-based, public health approach of the Fetal and Infant Mortality Review process (FIMR). The FIMR model is a systematic approach to understanding system gaps and obtaining insights into the factors that resulted in homelessness and ultimately death. This article reports on the process to develop this unique multi-agency, police-led review of homeless deaths in Indianapolis, Indiana and resulting recommendations for action to decrease these deaths.
\end{abstract}

Key Words: Homelessness; Death reviews; Mortality reviews; Cross-sector collaboration; FIMR 


\section{Introduction}

The Indianapolis Metropolitan Police Department (IMPD) has taken an innovative and progressive approach to working with the homeless population in Indianapolis. In 2011, IMPD created the Homelessness and Panhandling Unit as another specialty unit addressing a specific issue, just like homicides or auto thefts. The Homelessness and Panhandling Unit has established cross-system partnerships in an effort to keep homeless individuals out of the criminal justice system when possible and to connect them with services as appropriate (Hipple, 2016). As a result, they are extremely engaged with the chronically homeless in Indianapolis.

Nevertheless, law enforcement is historically reactive in nature. In March 2015, two fishermen found partially mummified human remains under a bridge near downtown Indianapolis. The Homelessness and Panhandling Unit regularly assists the Marion County Coroner's Office with initial identification in such cases and locating the deceased homeless individuals' next of kin. In this case, there were indications that the remains were those of a homeless individual. The Homelessness and Panhandling Unit and its existing agency partners were shocked when no one knew this individual even after he was positively identified. This sentinel event led to a considerable discussion with the question: if this deceased individual was unknown to the Homelessness and Panhandling Unit and no one from the outreach agencies had contact with him, were there other deceased homeless individuals they did not know about? In fact, this event confirmed there was no official count of homeless individual deaths in the Indianapolis and no easy way of creating one.

Indianapolis, Indiana, located in Marion County, spans 361 square miles and had an estimated population of 848,788 people in 2014 (United States Census Bureau, 2015). Point-inTime counts for 2015 accounted for 1,666 homeless individuals. Point-in-Time counts are known to be undercounts and research suggests the actual number of individuals experiencing 
homelessness at any given time is three to five times the point-in-time counts (Sankari \& Littlepage, 2015). The lack of knowledge regarding homeless deaths occurring in Indianapolis, a desire to understand how and why homeless individuals were dying, and the sentinel event death in March 2015 led the IMPD Homelessness and Panhandling Unit to partner with a local researcher. The goal of this partnership was and still is to collect data on homeless deaths and determine why these deaths occurred. A detailed review of homeless death cases seemed logical.

The police were most familiar with a crime incident review process (Klofas et al., 2006) but their approach needed modification because homeless deaths are not generally related to a specific crime. The approach they adapted is modeled after the Fetal and Infant Mortality Review process (FIMR). The FIMR process incorporates core public health functions that are applicable to complex health issues, such as the death of a fetus, infant, or homeless person. The environment and health of individuals are assessed based on multiple data sources and policy recommendations are made to prevent future deaths. The FIMR death review process continues to monitor and modify policy implementation. This article reports on the police-led FIMR process and its application to examining homeless deaths in Indianapolis, and resulting recommendations for action to decrease these deaths.

\section{Incident/Death Review Process and FIMR}

Incident reviews are not new to the criminal justice system (Azrael, Braga, \& O'Brien, 2013; Braga, Kennedy, Waring, \& Piehl, 2001; Hipple, McGarrell, O’Brien, \& Huebner, 2016; Klofas et al., 2006; O'Brien, Woods, \& Cisler, 2007) however, such law enforcement-led incident reviews most often focus on specific crimes such as homicides, non-fatal shootings, and domestic violence homicides that are within the police jurisdiction. Homelessness in itself is not a crime. And, the general consensus among agencies that worked with the homeless in 
Indianapolis, including law enforcement, is that the majority of homeless deaths are not the outcome of a criminal act, that is, not resulting from homicides, though this consensus was not based on any official data.

Homeless death reviews are occurring in other jurisdictions in the United States such as Philadelphia, Pennsylvania (Hoffman, Maguire, Cancellier, \& Cherington, 2012) and Sacramento County, California (Erienbusch, 2014). What makes the review process in Indianapolis unique is that the police are taking the lead and are using a public health approach. In addition to hosting the review meetings, IMPD sought an independent researcher to assist with data collection and analysis as well as facilitate the review meetings. The Homelessness and Panhandling Unit invited representatives from many of its existing partners to attend an informational meeting about the review process. They chose to model their reviews after the FIMR process because there was not focused on a specific crime type.

FIMR is an evidence-based, action-oriented community process that continually assesses, monitors, and works to improve service systems and community resources for women, infants, and families (Fetal and Infant Mortality Review Manual: A Guide for Communities (2nd Edition), 2008). The process engages a multi-disciplinary case review team to review deidentified infant and fetal deaths. The FIMR process includes a maternal interview for a consumer perspective on why the death occurred. The case review includes medical, social, and environmental factors about the death and therefore the case review team typically includes health care providers, obstetricians, social workers, mental health professionals, and health department staff. Based on these reviews, the case review team makes recommendations to improve any system gaps the team discovered. A community action team is then assembled to takes these recommendations into action. The community action team is usually comprised of 
community members, elected officials, and other key leaders who can implement change. The team works with at-risk families, and other partner agencies in the region to implement and develop street-level outreach activities.

The FIMR process is a standardized approach used to examine various morbidities and mortalities, i.e., elimination of mother-to-child transmission of HIV (Nesheim et al., 2012), maternal mortality, and maternal hospitalization for influenza. Research shows FIMR is an evidence based, effective perinatal systems intervention (Koontz, Buckley, \& Ruderman, 2004; McDonnell, Strobino, Baldwin, Grason, \& Misra, 2004). In Indianapolis, the FIMR process provides a systematic method to obtain information about homeless deaths beyond official police and outreach records. FIMR includes consumer/family interviews to determine their perspective on factors that may have contributed to the individual's life and death. Data sources include death certificate, police records, hospital records, information from homeless outreach program. Thus, the review process yields valuable information about social and environmental aspects of the case. Indianapolis partners concerned with homeless deaths supported using the FIMR process to examine causes and preventability of homeless deaths as it added in components not part of the traditional crime incident review (Fetal and Infant Mortality Review Manual: A Guide for Communities (2nd Edition), 2008).

\section{Homeless Death Reviews}

Research on homeless populations has demonstrated that homelessness in itself is an independent risk factor for death (Barrow, Herman, Córdova, \& Struening, 1999; Morrison, 2009). Mortality rates among homeless shelter residents in New York City were found to be four times higher than those of the general population (Barrow et al., 1999). A history of drug and alcohol use and abuse only increased the risk of death for homeless individuals (Barrow et al., 
1999; Cheung \& Hwang, 2004; McCartney et al., 2010; Morrison, 2009). This kind of information was not available about the homeless population in Indianapolis even though the Homelessness and Panhandling Unit maintained relationships with many agencies that worked with the local homeless population through an already existing case conferencing group (Hipple, 2016). This group, known as Community Outreach Taskforce or COT Force, served as the starting point for the case review team. Some but not all became case review team members in addition to others they recruited from outside of COT Force.

\section{Data Collection}

As a first step, the case review team compiled available data on homeless deaths occurring in Marion County. For this phase, partnering with a researcher proved to be invaluable. While the case review team was comprised of multidisciplinary experts working with the homeless population, they were not equipped to collect or maintain a single data source on homeless deaths. It was also important to the case review team that the review and recommendations be data-driven, and partnering with a researcher would help achieve this goal. Evidence from the field of criminal justice supports multi-agency partnerships and the integration of research for helping law enforcement achieve their problem-solving and crime reduction goals (Braga et al., 2001; McGarrell, Corsaro, Hipple, \& Bynum, 2010; Piehl, Kennedy, \& Braga, 2000; Roehl et al., 2008).

As previously mentioned, there was no systematic data collection process in place for collecting information on the deaths of homeless individuals and the process proved difficult and cumbersome to start. However, the City of Indianapolis does participate in the National Homeless Persons' Memorial Day. The 2014 and 2015 memorial lists and available Coroner's records were the starting point for data collection. The compiled list includes decedents who had 
been homeless at any time. And while the Memorial List definition of homeless was very broad, the case review team believed it was important not to be limited by the HUD definition of homeless because relevant cases would be missed. The Indianapolis team agreed upon a working definition for inclusion on the decedent list: any individual who had been homeless within 12 months of his or her death. Individuals were added to and removed from the master decedent list as deemed appropriate by the agreed upon criteria.

Systematic data collection included information on both the deceased individuals and the circumstances of their deaths. Sources available from the Coroner's Office (if the death investigation resulted in a Coroner case) included field deputy reports, autopsy reports, and death certificates. The Marion County Health Department assisted with death certificates as well. Additionally, the researcher conducted open source searches to supplement the official data sources. Retrospective data collection proved to be difficult. The more recent the case the easier it was to both determine the decedent met the definition of homeless created by the case review team as well as gather information. These issues reinforced the need to collect as much information, as close to the time of death as possible.

Table 1 displays demographic information for 101 deceased homeless individuals from 2014 and 2015. The majority are white, non-Hispanic males with a mean age of 48 years at the time of death. While national death trends are not available, the race and gender findings are similar to those found in Philadelphia (Hoffman et al., 2012) and Sacramento County (Erienbusch, 2014). Almost 18 percent of the deceased were military veterans. Other variables of interest include marital status, education, exact cause of death, employment status, and place of birth but these data are limited for some decedents. These variables were captured from both official and non-official sources. 
Table 2 displays death incident details of interest. Month of death was bi-modal, with the majority occurring in November and January. Most deaths occurred on Thursdays and inside a location deemed a residence but not necessarily the residence of the deceased individual. Time of death is limited because most decedents were alone at the time of death and time until discovery varies. The case review team did not find the Manner of Death categories as dictated by the Centers for Disease Control and Prevention (CDC) particularly informative in the case of homeless deaths. That is, the information was not helpful in looking for system fixes or making recommendations for change. The six CDC categories are: Natural, Accident, Suicide, Homicide, Pending Investigation, and Could not be Determined. The majority of homeless individual deaths in Indianapolis were ruled either Natural or Accident. Like race and gender, these manner of death findings are similar to those found in Philadelphia (Hoffman et al., 2012) and Sacramento County (Erienbusch, 2014). As one of the medical members of the case review team asked: what is natural about a 48 -year-old man dying when the average life expectancy is in the 70s? Therefore, the case review team created a second manner of death category, which incorporates the cause of death (i.e., diseases, injuries, or complications that directly caused the death). The new categories relate deaths to alcohol and/or drug use and abuse, disease (unrelated to drug/alcohol abuse), and trauma (see Table 2). Cause of death was important to the case review team because an analysis of the arrests of homeless 'chronic consumers' in Indianapolis revealed that almost $70 \%$ of arrests were for alcohol related offenses such as Public Intoxication (Hipple, 2016). Additionally, as previously mentioned, studies have shown drug and alcohol use by homeless individuals increases the risk of death (Barrow et al., 1999; Cheung \& Hwang, 2004; McCartney et al., 2010; Morrison, 2009). 
Case review data will also include informal interviews with family and/or friends of the deceased (Panel 1). After consultation with the National FIMR, strategies to discuss the life and death of the deceased with homeless encampment neighbors and family members were identified. Like the FIMR process, these informal interviews will provide a consumer/family perspective on the deceased's situation and perspective on factors that may have led to homelessness and his/her death. Data from medical records, police reports and other sources will also be compared to consumer information. Neighbors and family members will be contacted within one to three weeks after notification of the death. The neighbor/family perspective is important to obtain insights into system gaps that led to the death. In local FIMR programs, experience has shown that mothers want to tell their story and prevent future deaths. While the interview is not a grief therapy session, it does provide an opportunity for families to have a voice and tell their story. Referral for services for family and friends is an additional component of the process. The protocols for the interviews in Indianapolis are currently being developed and piloted.

\section{Review Meetings}

While the data collection process proved fruitful and provided useful baseline data, the data lacked sufficient incident-level detail needed to inform prevention efforts. This kind of information could not be culled from official records. More often, the specific details surrounding a decedent, his or her life, and the days leading up to death would need to come street-level workers who had interacted with the decedent. The case review process would serve to supplement the available official data. The IMPD Homelessness and Panhandling Unit reached out to its street-level partners to participate in the homeless death case review team meetings. Several informational meetings were held prior to actually reviewing cases to consider 
issues affecting productive reviews and information dissemination and discussion, such as the Health Insurance Portability and Accountability Act (HIPAA) Privacy Rule and Human Subjects Protections. For example, because the case reviews would involve deceased individuals, by definition, it was not research involving human subjects. However, the HIPAA Privacy Rule protects the health information of deceased individuals for 50 years from the date of their death just as if the individual were alive, with a few exceptions. Most team members were associated with an organization where at least one if not both of these issues were applicable. These issues were not difficult to resolve through the proper channels, however it did take time.

Prior to the review, case review team members with direct knowledge of the deceased person provide insight that enhances existing information from official records. Each represented agency is asked to provide agency relevant information ahead of time - most of which comes from official agency records. For example, the Homelessness and Panhandling Unit searches the police records management system for any incident reports involving the deceased. The prosecutor's office searches for criminal histories and active court cases. Probation checks the deceased's probation status during the year prior to death. Outside of law enforcement, meeting participants search for outreach contacts, EMS transports, emergency department visits, medical and mental health history, housing information, veteran status, and any relevant details about the deceased's life and death. The researcher then prepares a summary document for each case that is made available to team members prior to the review. During the reviews, team members provide information not available from official sources, such as information about the death incident itself, social engagement including the last time the decedent was physically seen by a team member, whether or not the decedent was in a relationship, keeps pets, has official or unofficial income, etcetera. These discussions provide additional information about the 
relationships and life of the deceased not captured in official records and thus facilitates the identification gaps in service while not assigning blame for the death. The researcher acts as a facilitator as well as a captures the detailed discussion.

\section{Recommendations for Action}

The FIMR process is driven by the goals of improving system services and community resources for women, infants, and families. Action-oriented recommendations are a critical part of meeting those goals. The homeless death case review team has met six times and reviewed 12 cases. And while each case is inherently different, the review process has revealed some themes across cases and the group has begun developing a list of actionable recommendations. Some of these recommendations can be addressed at the case review team level; others need to be elevated to the community action team.

First, the baseline data revealed that a common cause of death is exposure (considered accidental). In Indianapolis, the mid-western winters can be harsh. Outreach agencies have protocols in place to protect the privacy of their homeless clients as well as the safety of the outreach worker. For example, they do not go into tents without permission (i.e., the tent is treated like a house and an outreach worker would not enter a house without the owner's/occupant's permission). The reviews confirmed that during extreme weather emergencies, outreach workers felt hampered by this protocol. Furthermore, the reviews also revealed that outreach workers are often times told about individuals who will not come into shelters during extreme weather for one reason or another and the most logical place to look for that individual would be where they reside (i.e., his or her tent). Clearly situations like these have proved deadly for some. The homeless death case review team identified some modifications of protocols during extreme cold weather situations that may save lives. Notably, outreach 
personnel and the Homelessness and Panhandling Unit will be more assertive in locating homeless individuals during weather emergencies, such as looking into tents for vulnerable individuals when regular protocol would not allow this. Related, case review team members also reported that there is a culture for the homeless of 'not going in' or 'you're a sucker if you go in' during extreme cold weather. The case review team discussed ways to respectfully provide outreach including, 'mercy arrests,' immediate detentions, or other creative options by IMPD to shelter vulnerable individuals temporarily, to save lives. These proposed changes are similar to changes made by Philadelphia in 1987 in an effort to prevent cold weather homeless individual deaths. "Code Blue" sets a temperature/wind chill that triggers additional shelter beds, expanded street outreach and other actions. In 2009-2010, hypothermia was the primary cause of death in less than six percent of cases in Philadelphia (Hoffman et al., 2012).

Second, while official data easily confirmed emergency department 'frequent fliers' or 'chronic consumers' who are homeless, the case review team identified the need for better coordination between area hospitals and outreach workers. It is common for one of the large metropolitan hospitals to notify outreach workers when a homeless 'frequent fliers' or 'chronic consumers' presents at their emergency department. Outreach workers then visit that individual to try and engage him or her individuals in services. However, the reviews revealed that notification was not always consistent within or across area hospitals. This inter-agency communication between the hospitals and outreach needs to be expanded to all area hospitals located both downtown and outside of the city center to assure that homeless have access to available services.

Third, two of the twelve cases reviewed were situations where the client had agreed to leave the streets and go into detox. Anyone who works with the homeless population will agree 
that this is a very important milestone for both the homeless individual and those who work with him or her. The ideal situation is to 'take them when they are ready.' However, due to the lack of inpatient detox beds in Indianapolis, this rarely happens. Detox clients must first meet for an evaluation and then be scheduled to go into detox. One homeless individual had already been approved for a detox bed but died before the bed was available. The homeless individual had a scheduled date to enter detox but died three days prior. Both deaths were directly related to alcohol consumption. In both these situations, the case review team felt that if a detox bed had been available at the time the client was ready, the death would have been prevented. Detox facility bed availability is an issue that will need to be elevated to the community action team as this is not an issue the review team can or should work to resolve. Death review team members brought these details forward at the review and now they can be put forth in the form of a community-level recommendation which may prevent similar situations that have the potential to be fatal.

The establishment of the community action team is in the planning stages and key to the sustainability of this review process. The community action team is charged with developing new and creative solutions to improve services and resources for the homeless. In addition to law enforcement and outreach programs, key participation is needed from the Veteran's Administration (17.7\% of deaths), health department, hospitals and elected officials. These individuals have ability to garner the resources to address the system gaps identified in the review process. The case review team will provide specific recommendations that the community action team can implement to decrease homeless deaths in the community such as increasing the number of available detox beds. Commonly, the community wants solutions to the problems of the homeless but the right people are not at the table to solve the problems. The 
sponsor of the community action team should be an important and lead agency. For example, in Baltimore City, Maryland, the FIMR community action team is in the Mayor's office and it provides a high level of visibility and access to city agencies. This assures maximum impact for community action and commitment.

At the outset, the homeless death case review team outlined four goals: prevent future deaths of homeless individuals; create a better flow of information; compile accurate data on homeless individual deaths; and create actionable recommendations. They have made significant strides towards achieving these goals in a relatively short period of time. The benefits of a public health approach like FIMR to addressing homeless deaths are clear as official data simply do not tell the complete story and do not provide clear, actionable recommendations for prevention. However, one unanticipated benefit that resulted from the death reviews is that, in some cases, the partners determined that they did do everything possible to assist the deceased individual. That is, the system operated as it should. So, not every case is a sentinel event where gaps occurred and changes need to be made. For front line workers that often see the worst, this is an important, often overlooked, benefit on the review process.

\section{Conclusion}

The year 2016 started ominously for the homeless population in Indianapolis, Indiana. On January 1st, New Year's Day, two homeless individuals died. One individual was found by a friend at a known homeless encampment; the other died at a local shelter. By the sixth day of January, a total of four homeless individuals had died. Ranked one of the ten most violent cities in the United States in 2014 (Federal Bureau of Investigation, 2015), Indianapolis did not record its first criminal homicide until January 4, 2016. Homeless individuals, while omnipresent in most metropolitan areas, are often invisible. Their deaths are no different. 
A recent article in the British Journal of Medicine (BMJ) discusses the limitations of the death certificate - some of which are noted earlier in this report. Specifically, death certificates lack a place to indicate the human and social factors that led to death. The article focuses on medical errors and their contribution to deaths in the United States (Makary \& Daniel, 2016). While homelessness may not contribute to death in the same magnitude as medical errors, the death reviews discussed here, as well as other existing research, would suggest it is a contributing factor like medical errors and should be considered as such.

Homelessness is a complex problem for many of our communities with insufficient data. While providing housing is a long-term issue, preventing deaths is not. Homeless person death reviews are demonstrating that there are steps that can be taken to prevent needless deaths in this population. The FIMR process provides a systematic approach to understanding system gaps, provides insights into the factors that resulted in homelessness and ultimately death.

Additionally, the police can lead these efforts as part of a comprehensive problem solving effort. In summary, the police-led FIMR process for addressing homelessness in Indianapolis is an innovative approach to address this public health problem and will provide a structure for community solutions. 


\section{References}

Azrael, D., Braga, A. A., \& O'Brien, M. E. (2013). Developing the capacity to understand and prevent homicide: An evaluation of the Milwaukee Homicide Review Commission. Boston, MA: Harvard School of Public Health.

Barrow, S. M., Herman, D. B., Córdova, P., \& Struening, E. L. (1999). Mortality Among Homeless Shelter Residents in New York City. American Journal of Public Health, 89(4), 529-534. doi:10.2105/AJPH.89.4.529

Braga, A. A., Kennedy, D. M., Waring, E. J., \& Piehl, A. M. (2001). Problem-oriented policing, deterrence, and youth violence: An evaluation of Boston's Operation Ceasefire. Journal of Research in Crime and Delinquency, 38(3), 195-225. doi:10.1177/0022427801038003001

Cheung, A. M., \& Hwang, S. W. (2004). Risk of death among homeless women: A cohort study and review of the literature. Canadian Medical Association Journal, 170(8), 1243-1247. doi:10.1503/cmaj.1031167

Erienbusch, B. (2014). Sacramento County Homeless Deaths Report: 2002 - 2013 \& a 2014 update. Retrieved from Sacramento, CA:

Federal Bureau of Investigation. (2015). Crime in the United States, 2014. Retrieved from https://www.fbi.gov/about-us/cjis/ucr/crime-in-the-u.s/2012/crime-in-the-u.s.2012/violent-crime/aggravated-assault

Fetal and Infant Mortality Review Manual: A Guide for Communities (2nd Edition). (2008). Washington, DC: American College of Obstetricians and Gynecologists.

Hipple, N. K. (2016). Policing and homelessness: Using partnerships to address a cross system issue. Policing: A Journal of Policy and Practice. doi:10.1093/police/paw010

Hipple, N. K., McGarrell, E. F., O’Brien, M., \& Huebner, B. M. (2016). Gun crime incident reviews as a strategy for enhancing problem solving and information sharing. Journal of Crime and Justice, 1-18. doi:10.1080/0735648X.2016.1155303

Hoffman, R., Maguire, M., Cancellier, R., \& Cherington, M. (2012). City of Philadelphia Homeless Death Review: 2009-2010 Report. Retrieved from Philadelphia, PA:

Klofas, J. M., Hipple, N. K., McDevitt, J., Bynum, T. S., McGarrell, E. F., \& Decker, S. H. (2006). Project Safe Neighborhoods: Strategic interventions crime incident reviews: Case Study 3 Project Safe Neighborhoods: Strategic Interventions. Washington, DC: U.S. Department of Justice, Office of Justice Programs.

Koontz, A. M., Buckley, K. A., \& Ruderman, M. (2004). The evolution of fetal and infant mortality review as a public health strategy. Maternal and child health journal, 8(4), 195 203. doi:10.1023/B:MACI.0000047418.14086.fc

Makary, M. A., \& Daniel, M. (2016). Medical error-the third leading cause of death in the US. BMJ, 353, c2369. doi:10.1136/bmj.i2139

McCartney, G., Thomas, S., Thomson, H., Scott, J., Hamilton, V., Hanlon, P., . . Bond, L. (2010). The health and socioeconomic impacts of major multi-sport events: Systematic review (1978-2008). British Medical Journal, 340, c2369. doi:10.1136/bmj.c2369

McDonnell, K. A., Strobino, D. M., Baldwin, K. M., Grason, H., \& Misra, D. P. (2004). Comparison of FIMR programs with other perinatal systems initiatives. Maternal \& Child Health Journal, 8(4), 231-238.

McGarrell, E. F., Corsaro, N., Hipple, N. K., \& Bynum, T. S. (2010). Project safe neighborhoods and violent crime trends in US cities: Assessing violent crime impact. Journal of Quantitative Criminology, 26(2), 165-190. doi:10.1007/s10940-010-9091-9 
Morrison, D. S. (2009). Homelessness as an independent risk factor for mortality: Results from a retrospective cohort study. International Journal of Epidemiology, 38(3), 877-883. doi:10.1093/ije/dyp160

Nesheim, S., Taylor, A., Lampe, M. A., Kilmarx, P. H., Fitz Harris, L., Whitmore, S., . . . Mermin, J. (2012). A Framework for elimination of perinatal transmission of HIV in the United States. Pediatrics, 130(4), 738-744. doi:10.1542/peds.2012-0194

O'Brien, M., Woods, L., \& Cisler, R. A. (2007). The Milwaukee Homicide Review Commission: An interagency collaborative process to reduce homicide. Wisconsin Medical Journal, 106(7), 385.

Piehl, A. M., Kennedy, D. M., \& Braga, A. A. (2000). Problem solving and youth violence: An evaluation of the boston gun project. American Law and Economics Review, 2(1), 58106.

Roehl, J. A., Rosenbaum, D. P., Costello, S. K., Coldren, J., Schuck, A., Kunard, L., \& Forde, D. (2008). Paving the way for Project Safe Neighborhoods: SACSI in 10 US Cities. Washington, DC: US Department of Justice, Office of Justice Programs, National Institute of Justice.

Sankari, A., \& Littlepage, L. (2015). Many Families in Indiana Not Able to Find Shelter. Indianapolis, IN: Indiana University Public Policy Institute.

United States Census Bureau. (2015). QuickFacts Beta. Retrieved from http://www.census.gov/quickfacts/table/PST045214/1836003,00 
Table 1. Homeless decedent demographics

\begin{tabular}{|c|c|c|c|c|c|c|}
\hline \multirow[b]{2}{*}{ Variable } & \multicolumn{2}{|c|}{$\frac{2014}{(\mathrm{n}=63)}$} & \multicolumn{2}{|c|}{$\frac{2015}{(\mathrm{n}=38)}$} & \multicolumn{2}{|c|}{$\underline{\text { Total }}$} \\
\hline & $\underline{\mathrm{n}}$ & $\underline{\%}$ & $\underline{\mathrm{n}}$ & $\underline{\%}$ & $\underline{\mathrm{n}}$ & $\underline{\%}$ \\
\hline \multicolumn{7}{|l|}{ Race } \\
\hline Non-white & 20 & 31.7 & 9 & 23.7 & 29 & 28.7 \\
\hline White & 38 & 60.3 & 24 & 63.2 & 62 & 61.4 \\
\hline Missing & 5 & 7.9 & 5 & 13.2 & 10 & 9.9 \\
\hline \multicolumn{7}{|l|}{ Gender } \\
\hline Male & 36 & 57.1 & 26 & 68.4 & 62 & 61.4 \\
\hline Female & 13 & 20.6 & 7 & 18.4 & 20 & 19.8 \\
\hline Missing & 14 & 22.2 & 5 & 13.2 & 19 & 18.8 \\
\hline \multicolumn{7}{|l|}{ Age } \\
\hline Under 20 & 0 & 0.0 & 0 & 0.0 & 0 & 0.0 \\
\hline $20-29$ & 9 & 14.3 & 2 & 5.3 & 11 & 10.9 \\
\hline $30-39$ & 3 & 4.8 & 4 & 10.5 & 7 & 6.9 \\
\hline $40-49$ & 13 & 20.6 & 9 & 23.7 & 22 & 21.8 \\
\hline $50-59$ & 15 & 23.8 & 14 & 36.8 & 29 & 28.7 \\
\hline $60-69$ & 9 & 14.3 & 6 & 15.8 & 15 & 14.9 \\
\hline 70 or older & 0 & 0.0 & 0 & 0.0 & 0 & 0.0 \\
\hline \multirow[t]{2}{*}{ Missing } & 14 & 22.2 & 3 & 7.9 & 17 & 16.8 \\
\hline & M & SD & M & SD & M & SD \\
\hline Age & 47.0 & 12.0 & 49.3 & 10.0 & 48.0 & 11.2 \\
\hline \multicolumn{7}{|l|}{ Veteran } \\
\hline No & 34 & 54.0 & 21 & 55.3 & 55 & 54.5 \\
\hline Yes & 9 & 14.3 & 6 & 15.8 & 15 & 14.9 \\
\hline Unknown & 2 & 3.2 & 1 & 2.6 & 3 & 3.0 \\
\hline Missing & 18 & 28.6 & 10 & 26.3 & 28 & 27.7 \\
\hline
\end{tabular}


Table 2. Homeless death incident details

\begin{tabular}{|c|c|c|c|c|c|c|}
\hline \multirow[b]{2}{*}{ Manner of Death } & \multicolumn{2}{|c|}{$\frac{2014}{(\mathrm{n}=63)}$} & \multicolumn{2}{|c|}{$\frac{2015}{(\mathrm{n}=38)}$} & \multicolumn{2}{|c|}{$\underline{\text { Total }}$} \\
\hline & & & & & & \\
\hline Accident & 16 & 25.4 & 13 & 34.2 & 29 & 28.7 \\
\hline Homicide & 4 & 6.3 & 2 & 5.3 & 6 & 5.9 \\
\hline Natural* & 20 & 31.7 & 11 & 28.9 & 31 & 30.7 \\
\hline Suicide & 2 & 3.2 & 1 & 2.6 & 3 & 3.0 \\
\hline Undetermined & 3 & 4.8 & 1 & 2.6 & 4 & 4.0 \\
\hline \multicolumn{7}{|c|}{$\begin{array}{l}\text { * A natural death is caused solely by disease and/or other aging process } \\
\text { http://medical-dictionary.thefreedictionary.com/natural+death }\end{array}$} \\
\hline \multicolumn{7}{|l|}{ Cause of Death } \\
\hline Drug/Alcohol & 22 & 34.9 & 20 & 52.6 & 42 & 41.6 \\
\hline Trauma & 10 & 15.9 & 1 & 2.6 & 11 & 10.9 \\
\hline Disease & 10 & 1.9 & 4 & 10.5 & 14 & 13.9 \\
\hline Other & 2 & 3.2 & 0 & 0.0 & 2 & 2.0 \\
\hline Unknown & 1 & 1.6 & 1 & 2.6 & 2 & 2.0 \\
\hline Missing & 18 & 28.6 & 12 & 31.6 & 30 & 29.7 \\
\hline \multicolumn{7}{|c|}{ Month of the Year of Death } \\
\hline January & 10 & 15.9 & 3 & 7.9 & 13 & 12.9 \\
\hline February & 1 & 1.6 & 2 & 5.3 & 3 & 3.0 \\
\hline March & 1 & 1.6 & 1 & 2.6 & 2 & 2.0 \\
\hline April & 3 & 4.8 & 3 & 7.9 & 6 & 5.9 \\
\hline May & 3 & 4.8 & 1 & 2.6 & 4 & 4. \\
\hline June & 5 & 7.9 & 2 & 5.3 & 7 & 6.9 \\
\hline July & 2 & 3.2 & 4 & 10.5 & 6 & 5.9 \\
\hline August & 1 & 1.6 & 5 & 13.2 & 6 & 5.9 \\
\hline September & 7 & 11.1 & 5 & 13.2 & 12 & 11.9 \\
\hline October & 3 & 4.8 & 4 & 10.5 & 7 & 6.9 \\
\hline November & 8 & 12.7 & 5 & 13.2 & 13 & 12.9 \\
\hline December & 5 & 7.9 & 1 & 2.6 & 6 & 5.9 \\
\hline Missing & 14 & 22.2 & 2 & 5.3 & 16 & 15.8 \\
\hline
\end{tabular}

Day of the Week of Death

$\begin{array}{lcccccc}\text { Monday } & 5 & 7.9 & 8 & 21.1 & 13 & 12.9 \\ \text { Tuesday } & 7 & 11.1 & 2 & 5.3 & 9 & 8.9 \\ \text { Wednesday } & 6 & 9.5 & 4 & 10.5 & 10 & 9.9 \\ \text { Thursday } & 10 & 15.9 & 8 & 21.1 & 18 & 17.8 \\ \text { Friday } & 6 & 9.5 & 8 & 21.1 & 14 & 13.9\end{array}$




\begin{tabular}{lcccccc} 
Saturday & 7 & 11.1 & 4 & 10.5 & 11 & 10.9 \\
Sunday & 8 & 12.7 & 2 & 5.3 & 10 & 9.9 \\
\multicolumn{1}{c}{ Missing } & 14 & 22.2 & 2 & 5.3 & 16 & 15.8
\end{tabular}

\section{Temperatures (Days of Death)}

$\begin{array}{lcc}\text { Average High } & 57 & 70 \\ \text { Average Low } & 38 & 50 \\ \text { Minimum } & 6 & 20 \\ \text { Maximum } & 90 & 91\end{array}$




\section{CALL OUT BOX}

Panel 1: Family/Neighbor Questions

Tell me a little about your neighbor/family member.

What do you think led him/her to become homeless?

Why do you think the death occurred?

Is there service we could have provided to prevent the death?

What do you think we should do for others in a similar situation?

Is there something we can help you with now? 